\title{
A Review-on Analysis of Battery Lifetime Extension in Small-Scale Wind-Energy System using Super capacitors
}

\author{
Kaushal Kumar ${ }^{1}$, Dr. Yogesh Pahariya ${ }^{2}$ \\ M. Tech Scholar, (EE), RKDF Institute of Science \& Technology Bhopal, India ${ }^{1}$ \\ Director of RKDF, RKDF Institute of Science \& Technology Bhopal, India ${ }^{2}$
}

\begin{abstract}
Energy storage systems (ESSs) are enabling technologies for well-established and new applications such as power peak shaving, electric vehicles, integration of renewable energies, etc. With the increasing penetration of wind power into electric power grids, energy storage devices will be required to dynamically match the intermittency of wind energy. Energy-storage devices charge during low power demands and discharge during high power demands, acting as catalysts to provide energy boost. Batteries are the primary energy-storage devices in ground vehicles. Increasing the all-electric range of vehicles by $15 \%$ almost doubles the incremental cost of the energy-storage system. Due to the variable characteristics of renewable generation, batteries used in renewable-power systems can undergo many irregular, partial charge/discharge cycles. In turn, this can also have a detrimental effect on battery lifetime. Energy storage system in wind power system is required to deal with the difference of power between generator side and load side.
\end{abstract}

Keywords: Batteries, energy storage, super capacitors, wind power generation.

\section{INTRODUCTION}

Secondary batteries, super capacitors also known as "electrochemical double-layer capacitors" (EDLC), or "ultra capacitors," offer higher power density and increased cycle life (of the order of 106 cycles) but have a considerably lower energy density [3]. Super capacitors currently find use as short-term power buffers or secondary energy storage devices in renewable energy [4], [5], power systems [6], and transport applications. Wind Turbine generators (WTGs) are usually controlled to generate maximum electrical power from wind under normal wind conditions. However, because of the variations of the wind speed, the generated electrical power of a WTG is usually fluctuated. Currently, wind energy only provides about $1 \%-2 \%$ of the U.S.'s electricity supply. At such a penetration level, it is not necessary to require WTGs to participate in automatic generation control, unit commitment, or frequency regulation.

There are many forms of renewable energy readily available in the environment such as solar energy, mechanical energy, thermal energy, sound energy, wind power and so on. the active hybridization of batteries and super capacitors can yield an improvement in the overall energy storage system power handling. Wei et al. [11] have demonstrated that a battery-supercapacitor hybrid has lower battery costs, a general increase in battery life and higher overall system efficiency. To demonstrate the proposed system, an example low power $(<10 \mathrm{~kW})$ and low battery voltage $(<48 \mathrm{Vdc})$ wind energy conversion system. Energy storage system in wind power system is required to deal with the difference of power between generator side and load side. However, single storage element can not fulfill this goal ideally. Then, Hybrid energy storage system (HESS) is proposed that which combines two (or more) different kinds of storage elements has more superior performance than single storage element. In this present, a topology of hybrid energy storage system which consists of a battery and a super capacitor is used. Besides, a corresponding control strategy is proposed, which use moving average filter (MAF) to substitute traditional low pass filter to distinguish the DC and AC component of the imbalance power and deliver the DC part to the battery and absorb the AC part to the super capacitor. It makes full use of the energy storage elements and the battery life is extended.

The distribution of energy content of wind turbulence is described by the Van der Hoven wind speed spectrum [6]. This shows a clear distinction between long term wind speed fluctuations caused by the passage of weather systems (synoptic variations) or time of day (diurnal) and shorter fluctuations caused by turbulence [6,7]. There is a 'spectral gap' observed in the region between two hours and ten minutes where there is little energy between the synopticdiurnal region and the turbulent region. This gap is attributed to the lack of any physical process that could cause wind speed fluctuations in that range of frequency [6]. The turbulent region occurs over time periods ranging from $5 \mathrm{~s}$ up to $10 \mathrm{~min}$ with a peak at $1 \mathrm{~min}$. Turbulence causes fluctuations in power that have a negative effect on the quality of power delivered by a wind turbine, therefore these short term fluctuations are considered to be the most significant for the implementation of energy storage in wind energy systems [8]. As already mentioned, several ESS may be used to cover 
the electricity demand problems of numerous remote islands of Aegean Archipelago in collaboration with the existing APS and RES-based power stations (mainly wind parks and photovoltaic generators).

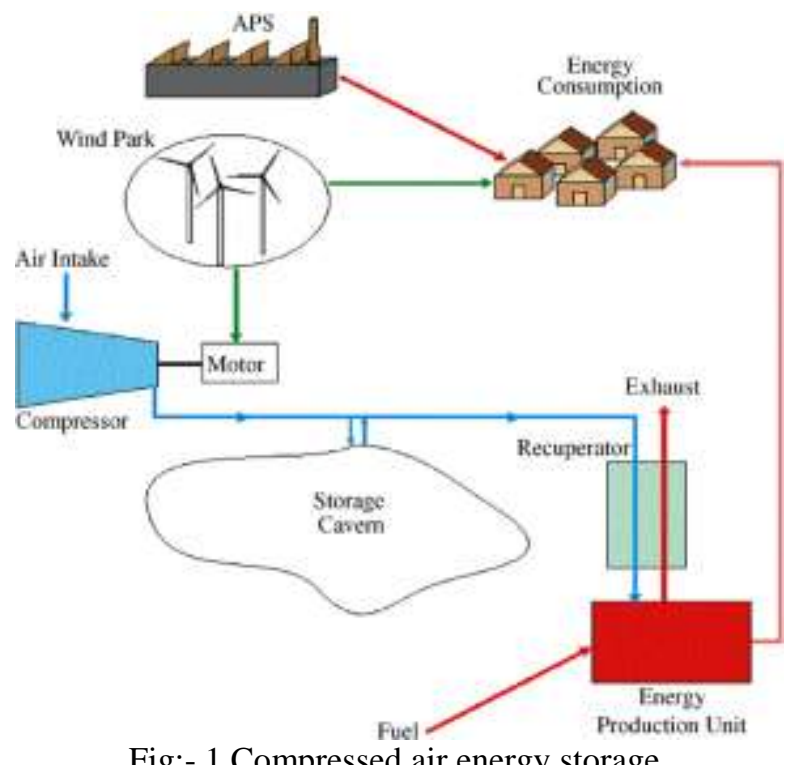

Fig:- 1 Compressed air energy storage.

Due to environmental and geopolitical concerns, there has been a renewed push to minimize the use of hydrocarbons for electric energy generation and transportation. These concerns have lead to the proliferation of electricity generation using both grid-tied and stand-alone renewable energy resources such as wind turbines and photovoltaic arrays. However, the intermittent nature of these resources introduces issues with system stability, reliability, and power quality. The issue of sporadic availability of renewable resources can be addressed by introducing energy storage systems (ESSs) to (partially) decouple energy generation from demand. In addition, the ESSs can be used to address the power quality issues by providing ancillary services to the grid. Large ESSs are routinely used alongside renewable generation such as wind to stabilize the power output.

Transportation electrification is seen as an effective way to substantially reduce the overall use of hydrocarbons. Electrified vehicles with plug-in capability contain an energy storage element that is capable of storing power from the grid.

\section{LITERATURE SURVEY}

Anthony M. Gee et. al. [1] “Analysis of Battery Lifetime Extension in a Small-Scale Wind-Energy System Using Super capacitors", In this study has investigated the use of super capacitors to improve expected battery life cycle over a representative weeklong power-profile typical of a small, remote-area wind-energy conversion system. The results show that by diverting transient power variations due to turbulence and short-term load variations to a supercapacitor module, battery life cycle can be quantifiably increased. It has also been shown that the battery current maxima can be significantly reduced using the proposed system. This has an unmodeled benefit in terms of potentially further increasing battery life as high-current cycling has been shown to increase battery failure rates [51]. The effect of increasing the low-pass filter time constant has been investigated and shown to further reduce battery cyclic wear and current maxima as the control-algorithm low-pass filter time-constant is increased. A versatile current controlled dc/dc converter design has been described, which was used to verify the feasibility and performance of the proposed system implementation. A detailed simulation strategy has been demonstrated, providing a means by which super capacitor control strategies can be evaluated in the context of an off-grid wind-energy conversion system.

Liyan Qu, et. al. [2] "Constant Power Control of DFIG Wind Turbines With Supercapacitor Energy Storage" in this paper has proposed a novel two-layer CPC scheme for a wind farm equipped with DFIG wind turbines. Each wind turbine is equipped with a super capacitor-based ESS, which is connected to the dc link of the DFIG through a twoquadrant dc/dc converter. The ESS serves as either a source or a sink of active power to control the generated active power of the DFIG wind turbine. Each individual DFIG wind turbine and its ESS are controlled by low-layer WTG controllers, which are coordinated by a high-layer WFSC to generate constant active power as required by or committed to the grid operator. Simulation studies have been carried out for a wind farm equipped with 15 DFIG wind turbines to verify the effectiveness of the proposed CPC scheme. Results have shown that the proposed CPC scheme enabled the wind farm to effectively participate in unit commitment and active power and frequency regulations of the 
grid. The proposed system and control scheme provides a solution to help achieve high levels of penetration of wind power into electric power grids.

Sergio Vazquez et. al. [3] "Energy Storage Systems for Transport and Grid Applications", in this ESSs are the key enabling technologies for transport and utility applications. In particular, the proliferation of energy storage will enable the integration and dispatch of renewable generation and will facilitate the emergence of smarter grids with less reliance on inefficient peak power plants. In the transportation sector, the emergence of viable onboard electric energy storage devices such as high-power and high-energy lithium ion batteries will enable the widespread adoption of plugin electric and HEVs which will also interact with the smart grids of the future. Mature storage technologies can be used in several applications, but in other situations, these technologies cannot fulfill with the application requirements. Thus,new storage systems have appeared, opening new challenges that have to be solved by the research community. Transport and utility applications operate with a wide range of time versus power storage requirements. The benefits obtained in transport and utility go from technical aspects to economic objectives.For instance, a reduction of $\mathrm{CO} 2$ emissions can be achieved by the use of EVs or increase of profits can be obtained with a load leveling application in a power transmission line. Anyway,the continuous development of the storage technologies and the evolution of their applications will motivate further research to solve the existing issues and improve the ESSs.

A Khaligh, et. al. [4] "Battery, Ultracapacitor, Fuel Cell, and Hybrid Energy Storage Systems for Electric, Hybrid Electric, Fuel Cell, and Plug-In Hybrid Electric Vehicles: State of the Art", in this The fuel economy and all-electric range (AER) of hybrid electric vehicles (HEVs) are highly dependent on the onboard energy-storage system (ESS) of the vehicle. Energy-storage devices charge during low power demands and discharge during high power demands, acting as catalysts to provide energy boost. Batteries are the primary energy-storage devices in ground vehicles. Increasing the AER of vehicles by $15 \%$ almost doubles the incremental cost of the ESS. This is due to the fact that the ESS of HEVs requires higher peak power while preserving high energy density. Ultracapacitors (UCs) are the options with higher power densities in comparison with batteries. A hybrid ESS composed of batteries, UCs, and/or fuel cells (FCs) could be a more appropriate option for advanced hybrid vehicular ESSs. This paper presents state-of-the-art energy-storage topologies for HEVs and plug-in HEVs (PHEVs). Battery, UC, and FC technologies are discussed and compared in this paper. In addition, various hybrid ESSs that combine two or more storage devices are addressed.

H Zhou, et. al. [5] "Composite Energy Storage System Involving Battery and Ultracapacitor With Dynamic Energy Management in Microgrid Applications", in this Renewable-energy-based microgrids are a better way of utilizing renewable power and reduce the usage of fossil fuels. Usage of energy storage becomes mandatory when such micro grids are used to supply quality power to the loads. Micro grids have two modes of operation, namely, grid-connected and islanding modes. During islanding mode, the main responsibility of the storage is to perform energy balance. During grid-connected mode, the goal is to prevent propagation of the renewable source intermittency and load fluctuations to the grid. Energy storage of a single type cannot perform all these jobs efficiently in a renewable powered micro grid. The intermittent nature of renewable energy sources like photovoltaic (PV) demands usage of storage with high energy density. At the same time, quick fluctuation of load demands storage with high power density. This paper proposes a composite energy storage system (CESS) that contains both high energy density storage battery and high power density storage ultra capacitor to meet the aforementioned requirements. The proposed power converter configuration and the energy management scheme can actively distribute the power demand among the different energy storages. Results are presented to show the feasibility of the proposed scheme.

Fangcheng Liu, et. al. [6] "A novel control strategy for hybrid energy storage system to relieve battery stress", in this presented Energy storage system in wind power system is required to deal with the difference of power between generator side and load side. However, single storage element cannot fulfil this goal ideally. Then, Hybrid energy storage system (HESS) is proposed that which combines two (or more) different kinds of storage elements has more superior performance than single storage element. In this paper, a topology of hybrid energy storage system which consists of a battery and a super capacitor is used. Besides, a corresponding control strategy is proposed, which use moving average filter (MAF) to substitute traditional low pass filter to distinguish the DC and AC component of the imbalance power and deliver the DC part to the battery and absorb the AC part to the super capacitor. It makes full use of the energy storage elements and the battery life is extended.

\section{III.METHOD}

All Due to turbulent wind power variations and short-term load variations, batteries can be expected to undergo frequent charge/discharge cycling in remote-area wind-power systems. The most common energy storage technology implemented in off-grid RE-membrane systems is lead-acid batteries [9-11], which are both relatively cheap and readily available [11]. Lead acid batteries are ideal for long-term energy storage due to their high energy density, efficiency $(85-90 \%)$ and low self discharge rate of $2 \%$ of rated capacity per month [10]. Super capacitors are energy storage devices that operate by building up positive and negative charge within an electrolytic solution, as opposed to the chemical reaction used in batteries. 


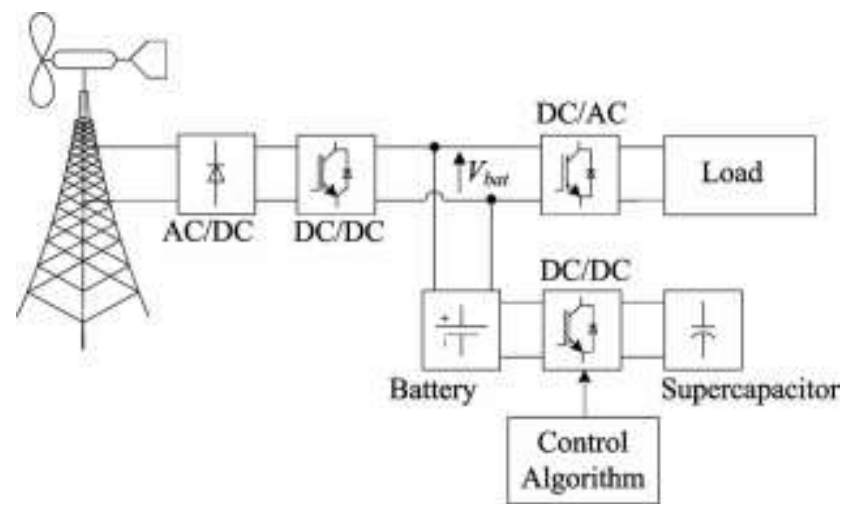

Fig. 1. Remote wind-energy conversion system configuration

They are also referred to as ultra capacitors or electrochemical double layer capacitors and are one of the most promising devices for buffering short term fluctuations from RE sources. While high power batteries such as lithiumion are more appropriate for longer periods of time $(10-15 \mathrm{~min})$ Super capacitors have been used in parallel with batteries to extend battery lifetime by reducing the number of charge/discharge cycles and buffering the peak currents in the battery. A synergy exists between both technologies where high power or current pulses can be met by the super capacitors while longer-term energy requirements are provided by the battery. There are also numerous examples of the application of super capacitors to large-scale grid-connected RE systems in order to smooth fluctuations, provide power during intermittency and re-start turbines when the grid fails. Experimental research using a $3 \mathrm{~kW}$ wind turbine simulator with power output fluctuations $(0-700 \mathrm{~W})$ demonstrated the ability of super capacitors to buffer wind fluctuations over short Periods of time (300 s). Supercapacitor banks:- For these experiments, modular (15 VDC) super capacitors (Maxwell BOOSTCAP BPAK0058 E015 B01, parameters shown in Table 1) were chosen to allow for expansion and experimentation with the optimum super capacitor bank size. These modules had the cells enclosed in durable packaging for safety, with integrated balancing electronics to ensure the cell-to-cell voltage was balanced when connected in series and the leakage current was minimized [24]. Within each super capacitor, the cells are stacked in series to increase their limited voltage range ( $1-3$ VDC) caused by low voltage stability of the electrolyte, and extend the useable voltage output which varies linearly according to the SOC [28]. The following design steps were used to size the super capacitor bank

1. The maximum voltage output from the wind turbine (Vmax $\sim 60$ VDC) determined the maximum charging voltage and therefore the size of the super capacitor bank based on the rated voltage of the super capacitor modules (VR). The number of super capacitor modules in series (Nseries) was calculated using

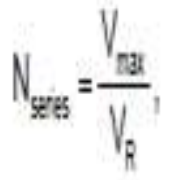

where Nseries was determined as four for this system.

2. The total amount of charge stored was determined by the capacitance of the supercapacitor bank, $\mathrm{C}_{\text {bank, }}$ Farads (F) as

$$
C_{\text {bank }}=C_{\text {module }} \times \frac{N_{\text {parallel }}}{N_{\text {senes }}} \text {, }
$$

Where $\mathrm{N}_{\text {series }}$ was four and the number of parallel rows $\left(\mathrm{N}_{\text {parallel }}\right)$ was varied from $1-3$ (therefore $\mathrm{C}_{\text {bank }}=14.5,29,43.5$ F) to increase the amount of system run-time and therefore determine the most appropriate size of energy storage for the system.

3. Two efficiency losses associated with super capacitors are the equivalent parallel resistance (EPR) and the equivalent series resistance (ESR). The EPR represents a current leakage path within the super capacitor that limits the long term capabilities of the device. This value was given as $1 \mathrm{~mA}$ by the manufacturer (Table 1) and was dependent upon the charge voltage and ambient temperature. Experiments showed the typical energy loss due to current leakage as $1-2 \%$ per day. The ESR is important for charge/discharge efficiency as it represents the material resistances of the electrode and electrolyte that result in internal heating. The ESR depends on the number of cells in series or parallel. Increasing cells in parallel reduces the resistance whereas more cells in series increases the resistance. The resistance of the supercapacitor bank (Rbank) was calculated using 


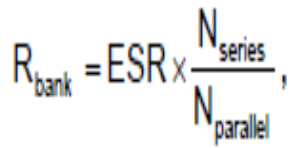

With the resistance of the $4 \times 1-4 \times 3$ supercapacitor banks (4 in series x 1, 2 or 3 rows in parallel) being 76, 38 and 25 $\mu \Omega$, respectively.

4. In order to get an indication of the amount of storage time $(\Delta \mathrm{t})$ available from the supercapacitor bank, the following equation was used

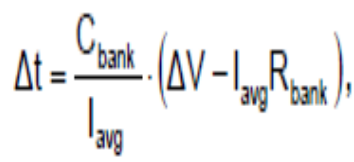

where $I_{\text {avg }}$ is the average discharge current and $\Delta V$ is the voltage difference between $V_{\max }(60$ VDC) and the minimum operating voltage (31.2 VDC). This equation could be used for charging or discharging. For an average discharge current of $5.3 \mathrm{~A}$, this would give a discharge time of $78 \mathrm{~s}$ for a $4 \times 1$ supercapacitor bank. As discussed earlier, this length of time would be ideal for improving the power quality from the wind turbine by buffering turbulent wind speed fluctuations that frequently occur at periods of about $1 \mathrm{~min}[6]$.

5. The energy $\left(\mathrm{W}_{\mathrm{h}}\right)$ stored in the supercapacitor bank (Figure 2) was proportional to the charge voltage (V) squared where

$$
E=\sqrt{2} \int_{\text {and }} W^{2}
$$

Therefore, it was possible to use $75 \%$ of the electrical energy stored in the Supercapacitor bank by discharging to half of its maximum voltage (60 VDC) [28]. Although supercapacitors have no lower operating threshold and can be discharged to zero volts, most DC/DC converters have a minimum voltage which limits the use of energy [24]. In this particular application the DC/DC converter limited the minimum voltage at $31.2 \mathrm{~V}_{\mathrm{DC}}$ which was equivalent to $27 \%$ minimum SOC (Equation6).

6. The SOC of the supercapacitor bank is a useful measure of the available energy as a percentage of the banks maximum energy capacity (Emax) [24], and was calculated as

$$
\mathrm{SOC}=\frac{\mathrm{E}_{\text {bank }}}{\mathrm{E}_{\mathrm{max}}} \cdot 100 \%,
$$

where the energy was calculated using Equation 5 with $60 \mathrm{VDC}$ for $\mathrm{E}_{\max }$ and the supercapacitor bank charge voltage for $E_{\text {bank }}$

\section{IV.CONCLUSION}

In this paper proposed has investigated the use of supercapacitors to improve expected battery life cycle over a representative weeklong power-profile typical of a small, remote-area wind-energy conversion system. The suitability of supercapacitors to expand the safe operating window of a wind-membrane system was examined in a systematic manner. The supercapacitors were able to provide sufficient energy during periods of no wind (intermittency) and enhance the power quality delivered to the membrane by absorbing turbulent wind (fluctuations). As a result, system shut-down and compromised permeate quality due to reduced TMP were avoided. The uses of supercapacitors to provide constant power resulted in a $40 \%$ increase in the average flux and $15 \%$ increase in permeate quality under intermittent operation over one hour. The improvements in the average flux and permeate quality under fluctuating conditions due to increased power quality were $85 \%$ and $40 \%$, respectively. While the SOC of the supercapacitor bank was above the minimum threshold value of $27 \%$, the membrane system operated as under steady-state conditions regardless of the wind speed and power output From the wind turbine.

\section{REFERENCES}

[1] Gavin L. Parka, Andrea I. Schäferb, Bryce S. Richardsa et. al. "Renewable energy powered membrane technology: Super capacitors for buffering resource fluctuations in a wind-powered membrane system for brackish water desalination”, Renewable Energy • February 2013. 
[2] J.K. Kaldellis , D. Zafirakis, K. Kavadias et. al. "Techno-economic comparison of energy storage systems for island autonomous electrical networks", Renewable and Sustainable Energy Reviews 2 November 2007.

[3] Raul Moraisa, Samuel G. Matosb, Miguel A. Fernandesb, Ant'onio L.G. Valentea, Salviano F.S.P. Soaresa,, P.J.S.G. Ferreirac, M.J.C.S. Reisa,et. al., "Sun, wind and water flow as energy supply for small stationary data acquisition platforms", computers and electronics in agriculture 64 ( 20008 ) 120-132.

[4] ETH Zurich et. al. "Multi-purpose technologies, lock-in and efficiency - Policy implications from the case of stationary electricity storage", London School of Economics and Political Science born 23.09.1984.

[5] Anthony M. Gee, Francis V. P. Robinson and Roderick W. Dunn, et. al. "Analysis of Battery Lifetime Extension in a Small-Scale Wind-Energy System Using Supercapacitors", IEEE Transactions on Energy Conversion, vol. 28, no. 1, march 2013.

[6] Jonathan W. Kimball, Brian T. Kuhn ,Robert S. Balog et. al. "A system design approach for unattended solar energy harvesting supply", IEEE Transactions on Power Electronics, vol. 24, no. 4, april 2009.

[7] Z.G. Wan, Y.K. Tan and C. Yuen et. al. "Review on Energy Harvesting and Energy Management for Sustainable Wireless Sensor Networks", SEPTEMBER 2011.

[8] J. Peinke, S. Barth, F. Böttcher, D. Heinemann, B. Lange, Turbulence, a challenging problem for wind energy, Physica A: Statistical Mechanics and its Applications, 338 (2004) 187-193.

[9] G. Petersen, S. Fries, J. Mohn, A. Muller, Wind and solar powered reverse osmosis desalination units - design, start up, operating experience, Desalination, 39 (1981) 125-135.

[10] D. Infield, Performance analysis of a small wind powered reverse osmosis plant, Solar Energy, 61 (1997) 415-421

[11] D. Weiner, D. Fisher, E.J. Moses, B. Katz, G. Meron, Operation experience of a solarand wind-powered desalination demonstration plant, Desalination, 137 (2001) 7-13. 